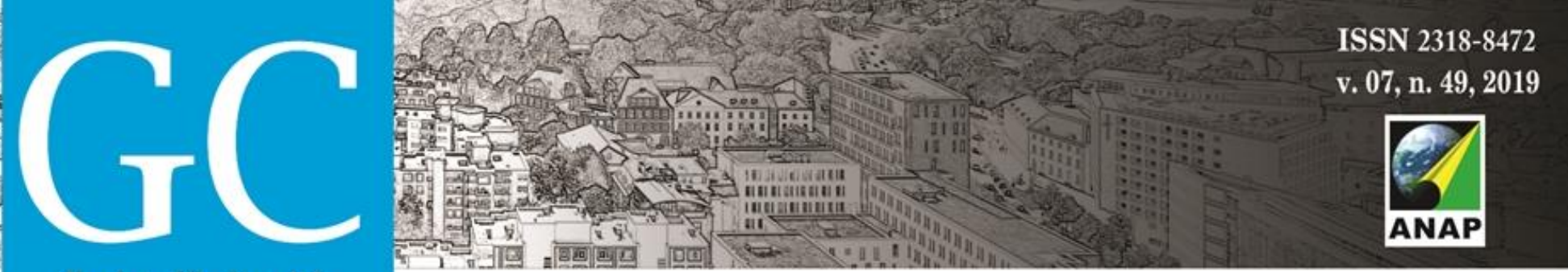

Revista Nacional de Gerenciamento de Cidades

\title{
Metodologia de Análise Urbana Tipo-Morfológica: Transformações Urbanas no Bairro Aparecida em Uberlândia-MG
}

Methodology of Urban Type-Morphological Analysis:

Urban transformations in the neighborhood Aparecida in Uberlândia-MG

Metodología de Análisis Morfológico Tipo Urbano:

Transformaciones urbanas en el barrio Aparecida en Uberlândia-MG

\author{
Glauco de Paula Cocozza \\ Professor Doutor, UFU, Brasil \\ glauco_cocozza@yahoo.com.br \\ Gabriela de Oliveira Bertuluci \\ Mestranda, UFU, Brasil \\ gabriela_bertuluci@hotmail.com
Bárbara Oliveira Silva
Mestranda, UFU, Brasil bos.arq@gmail.com \\ Schirley Crisitane de Oliveira Brandão \\ Mestranda, UFU, Brasil \\ schirleycobrandao@gmail.com
}




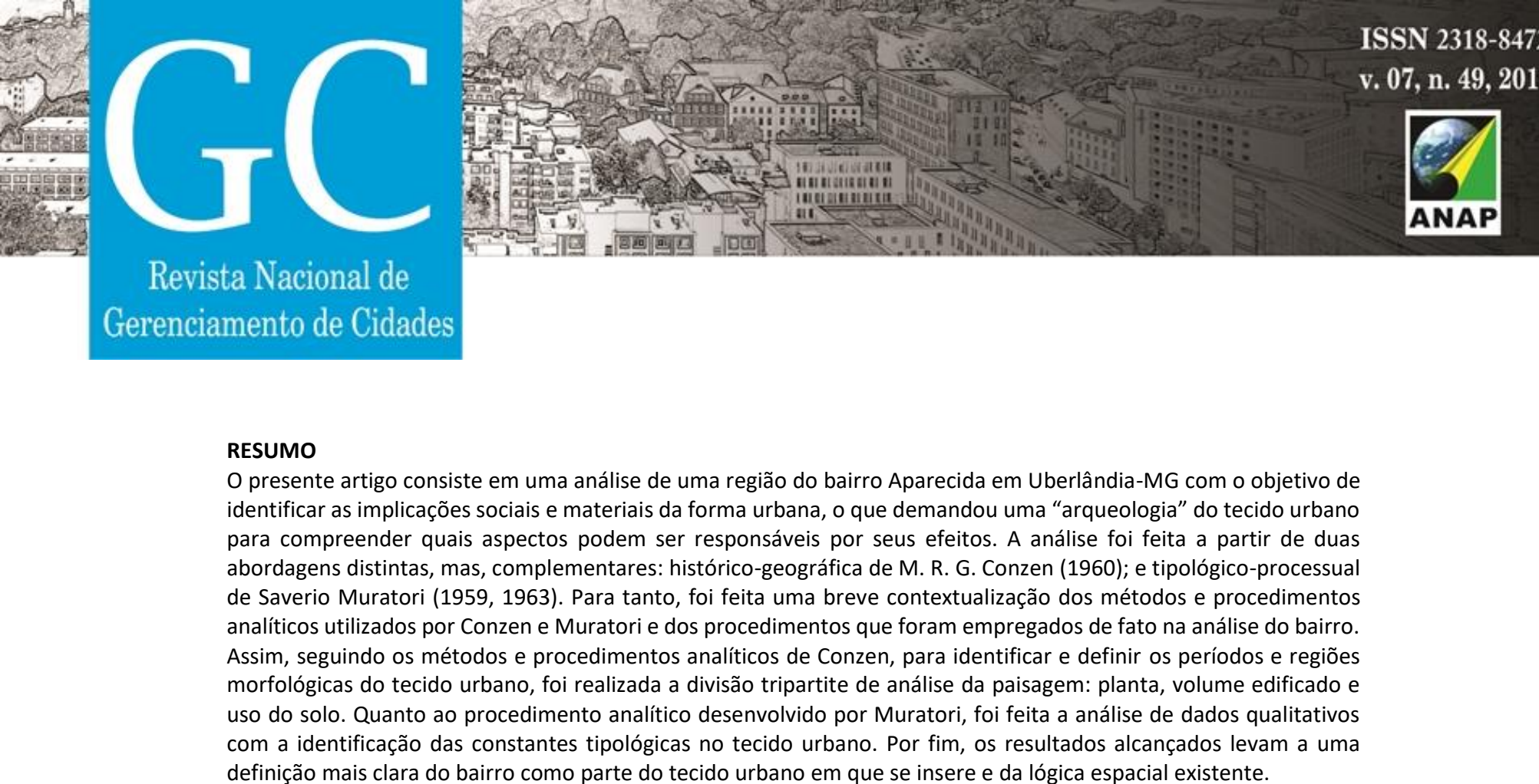

PALAVRAS-CHAVE: Forma Urbana; Transformações Urbanas; Tipo-morfologia

\section{ABSTRACT}

The present article consists of an analysis of a region of Aparecida neighborhood in Uberlândia-MG with the objective of identifying the social and material implications of the urban form, which demanded an "archeology" of the urban fabric to understand which aspects may be responsible for its effects. The analysis was made from two different but complementary approaches: historical-geographical of M. R. G. Conzen (1960); and typological-processual of Saverio Muratori $(1959,1963)$. For that, a brief contextualization of the analytical methods and procedures used by Conzen and Muratori and the procedures that were actually employed in the neighborhood analysis were made. Thus, following the methods and analytical procedures of Conzen, to identify and define the periods and morphological regions of the urban fabric, the tripartite division of landscape analysis was carried out: plant, volume and land use. As for the analytical procedure developed by Muratori, the analysis of qualitative data was done with the identification of the typological constants in the urban fabric. Finally, the results achieved lead to a clearer definition of the neighborhood as part of the urban fabric in which it is inserted and of the existing spatial logic.

KEYWORDS: Urban form; Urban Transformations; Type-morphological.

\section{RESUMEN}

El presente trabajo consiste en un análisis de una región del barrio Aparecida en Uberlândia-MG con el objetivo de identificar las implicaciones sociales y materiales de la forma urbana, que exigía una "arqueología" del tejido urbano para comprender qué aspectos pueden ser responsables por sus efectos. El análisis se realizó a partir de dos enfoques distintos pero complementarios: histórico-geográfico de M. R. G. Conzen (1960); y tipológico-procesal de Saverio Muratori $(1959,1963)$. Con este fin, se realizó una breve contextualización de los métodos y procedimientos analíticos utilizados por Conzen y Muratori y los procedimientos que realmente se utilizaron en el análisis del barrio. Por lo tanto, siguiendo los métodos y procedimientos analíticos de Conzen, para identificar y definir los períodos morfológicos y las regiones del tejido urbano, se realizó una división tripartita del análisis del paisaje: planta, volumen de construcción y uso del suelo. En cuanto al procedimiento analítico desarrollado por Muratori, se analizaron datos cualitativos con la identificación de constantes tipológicas en el tejido urbano. Finalmente, los resultados logrados conducen a una definición más clara del vecindario como parte de su tejido urbano y la lógica espacial existente.

PALABRAS CLAVE: Forma urbana; Transformaciones urbanas; Tipo de morfologia. 


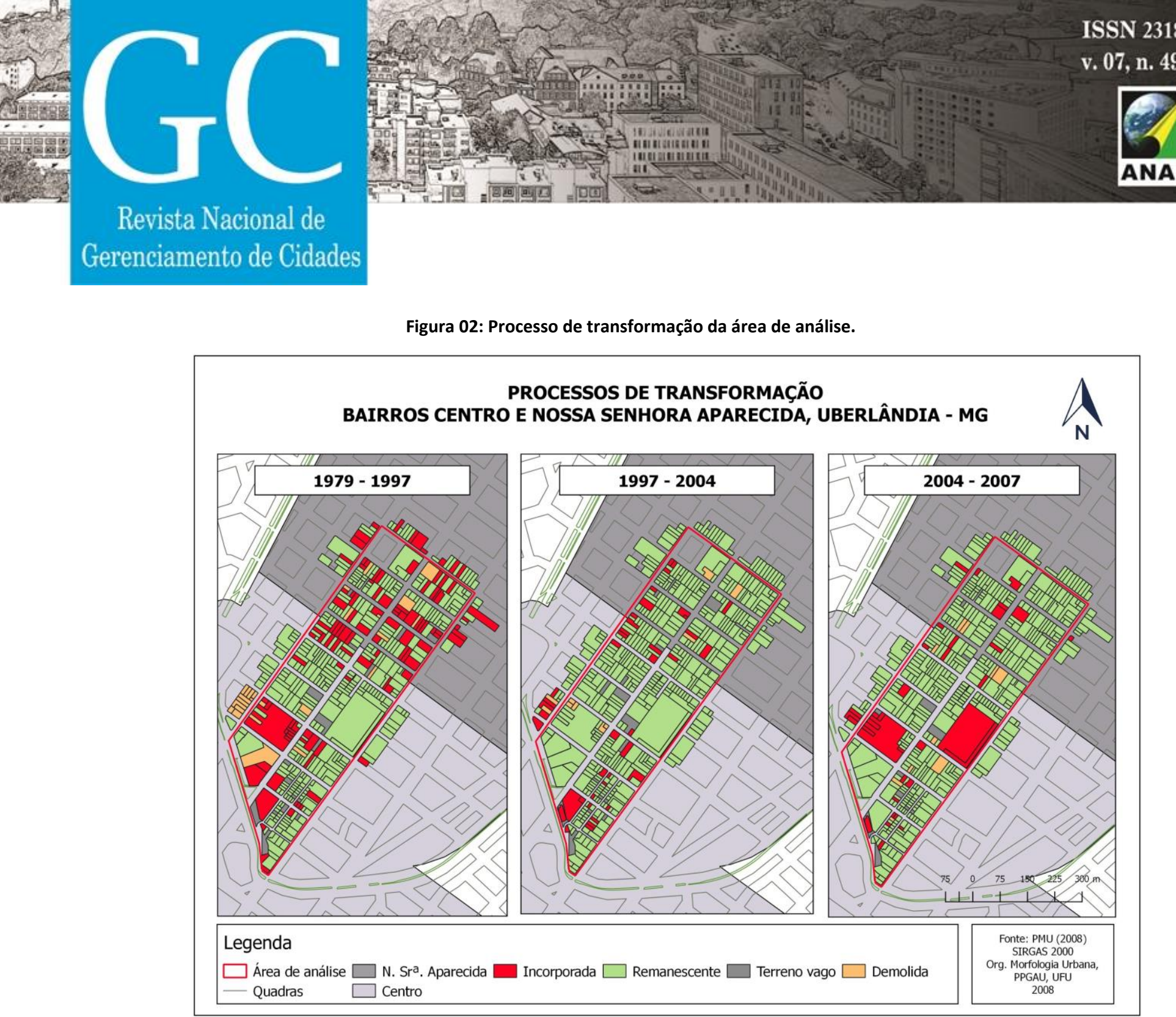

Fonte: Elaborado pelos alunos da disciplina Morfologia Urbana e Desenho da Cidade - PPGAU/UFU, 2018

A partir dessa análise, foi possível compreender que o principal processo de transformação da região, no recorte de tempo analisado, foi a substituição da Ferrovia Mogiana pela Praça Sérgio Pacheco e, posterior implantação do terminal central nessa. Essa mudança fez com que as ruas da região analisada (que estão lindeiras à praça), apesar de permanecerem com a mesma forma tivessem alteração de fluxo, passando de acesso restrito às residências para umas das avenidas de maior conexão da cidade, o que alterou completamente a sua dinâmica urbana.

Depois da análise das transformações morfológicas, foi desenvolvida uma série cartográfica composta de seis bases - Edificações, Lotes, Implantação, Volume Edificado, Uso do Solo e Regiões Morfológicas, que sintetizam a tipologias encontradas (Figura 3 e Figura 4). Importante salientar que a análise se concentrou na área delimitada, mas, as regiões morfológicas identificadas podem se estender para além da área de estudo. 



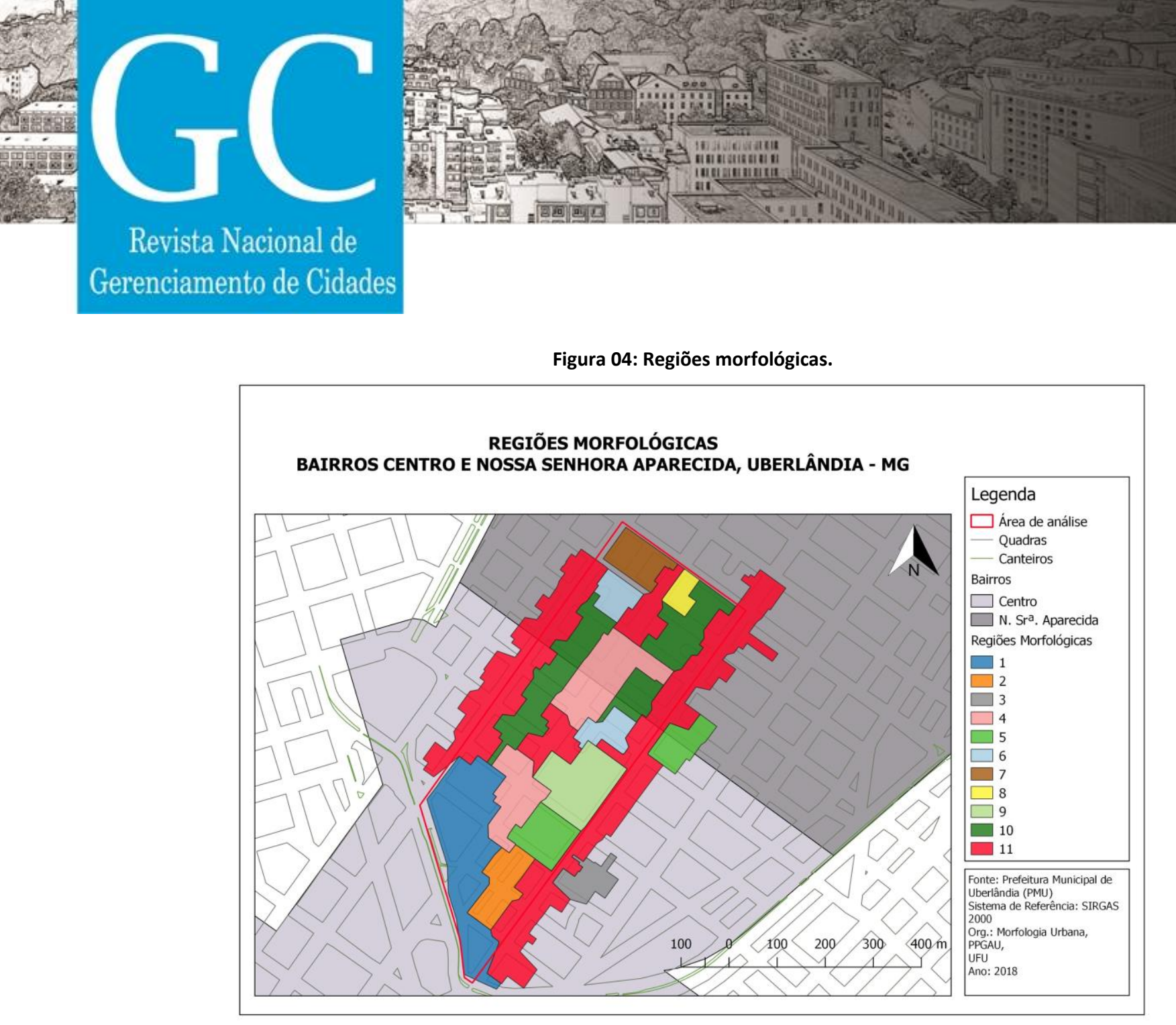

Fonte: Elaborado pelos alunos da disciplina Morfologia Urbana e Desenho da Cidade - PPGAU/UFU, 2018.

Nas duas primeiras bases, para analisar os lotes foi considerado sua forma - retangular, quadrangular, triangular, irregular, formato em " $\mathrm{L}$ "; e tamanho - pequenino (PP) - $<125 \mathrm{~m}^{2}$; pequeno $(P)-125<$ área $<250$, médio $(M)-250<$ área $<450$, grande $(G)-450<$ área $<1200 \mathrm{~m}^{2}$, muito grande (GG) - >1200 $\mathrm{m}^{2}$. Para analisar os edifícios foi considerada sua implantação no lote - através da planta de cheios e vazios; e seu gabarito. No caso da base do volume edificado foi considerada a relação da ocupação do solo com uso, sendo identificadas as categorias: edificação térrea sem afastamento, edificação térrea com afastamento, sobrado com afastamento, sobrado sem afastamento, galpão, edifício, estacionamento, especial. Por fim, a base de Uso do Solo considerou as categorias: residencial; comercial; serviço; uso misto; institucional; estacionamento.

A partir desse levantamento, foi possível identificar a lógica espacial da região, caracterizada, principalmente, por três fenômenos: área de influência da Praça Sérgio Pacheco, com concentração de galpões, serviços e comércio, caracterizada pela incorporação de elementos sob influência da antiga Estação Ferroviária; a permanência da característica de vila dos miolos de quadra, com predomínio de residências térreas e residenciais, porém com tendência a substituição do uso do solo, devido a posição de centralidade adquirida pelo bairro; e por fim, as avenidas Afonso Pena e Floriano Peixoto com concentração comercial e de serviços, fluxo intenso de veículos e adensamento construtivo. 


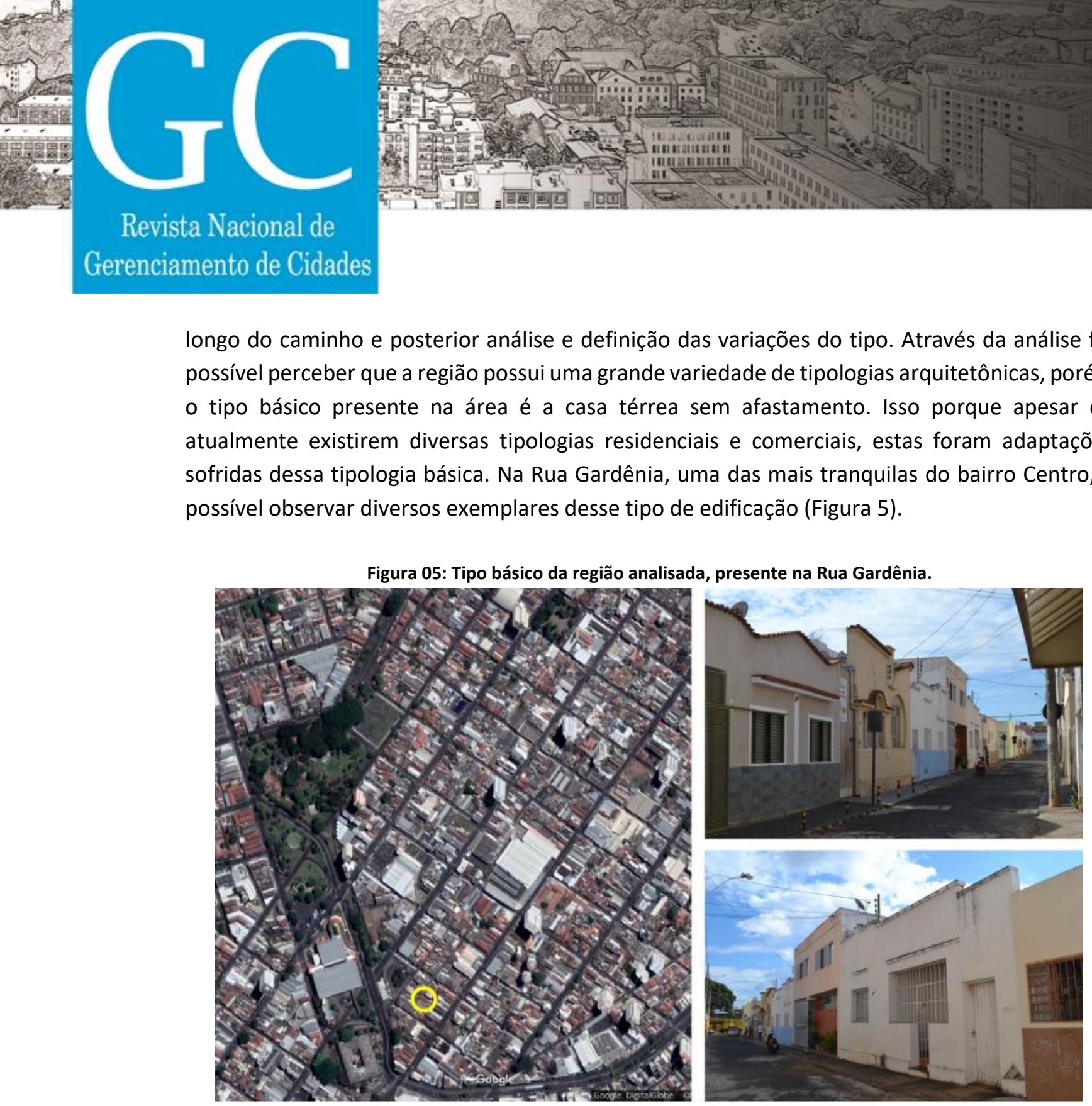

longo do caminho e posterior análise e definição das variações do tipo. Através da análise foi possível perceber que a região possui uma grande variedade de tipologias arquitetônicas, porém tipo básico presente na área é a casa térrea sem afastamento. Isso porque apesar de tualmente existirem diversas tipologias residenciais e comerciais, estas foram adaptações sofridas dessa tipologia básica. Na Rua Gardênia, uma das mais tranquilas do bairro Centro, é possível observar diversos exemplares desse tipo de edificação (Figura 5).

Fonte: Elaborada pelos autores, 2018

Na Figura 06 estão representadas as edificações que hoje abrigam pequenos comércios ou serviços. São modificações do tipo básico, onde uma residência térrea foi transformada em edifício comercial. Muitos destes estabelecimentos ocupam uma edificação que foi dividida, como por exemplo uma única casa que deu lugar a duas lojas. É possível observar na imagem o telhado das antigas casas, escondido pelas fachadas ocupadas pelas placas dos comércios. 


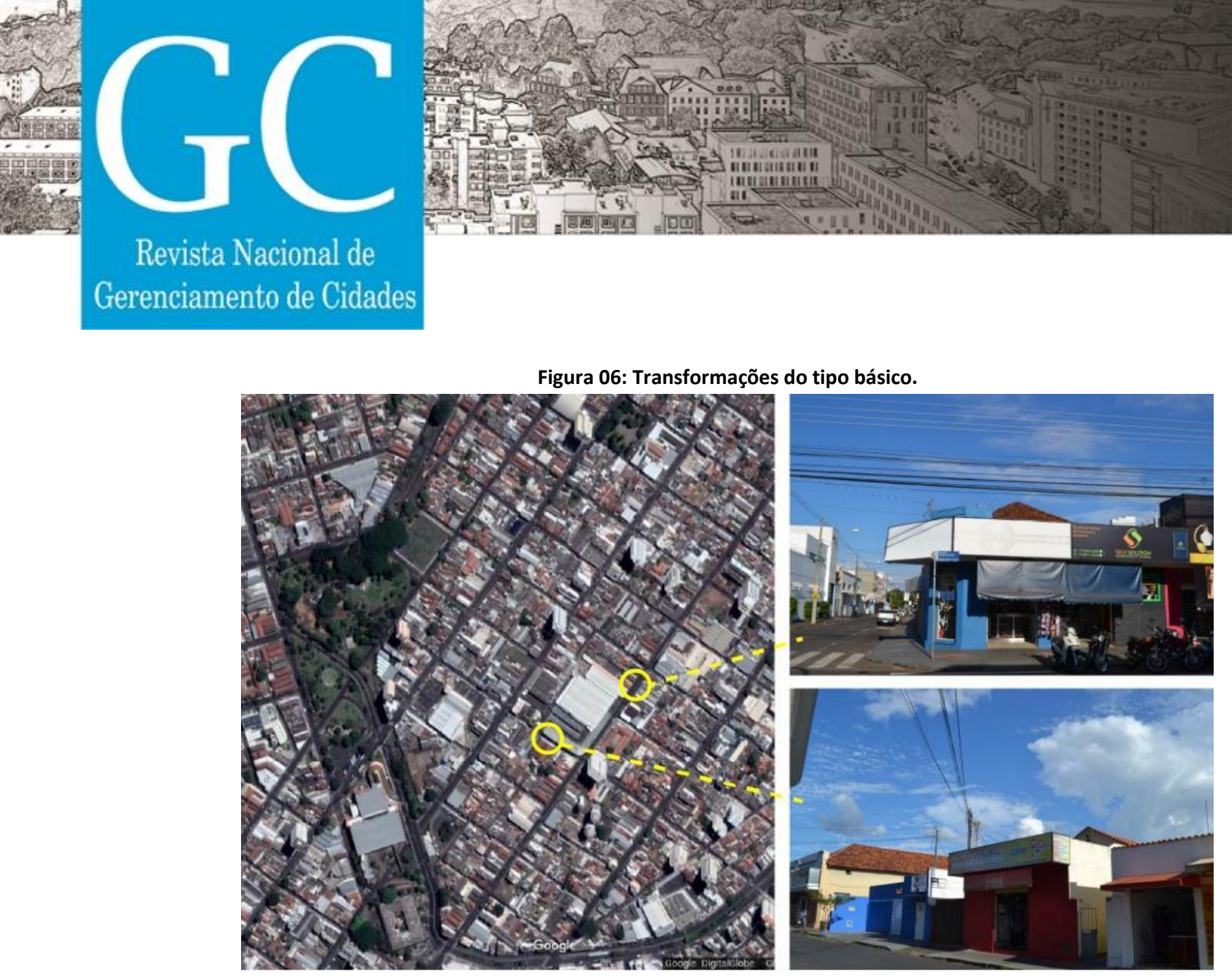

Fonte: Elaborada pelos autores, 2018.

Na Figura 07 observa-se que na região analisada existem alguns exemplares de uma tipologia residencial que se difere um pouco do tipo original. Esses edifícios são mais recentes e consistem em casas que possuem um grande afastamento frontal separado da calçada por um gradil. Essa tipologia é encontrada somente nos miolos de quadra, que possuem uma atmosfera completamente diferente da presente nas Av. Afonso Pena e Floriano Peixoto.

$\mathrm{Na}$ área estudada também existem outros tipos de casas térreas, que são gerados quando uma edificação mais antiga é completamente demolida e dá lugar à um edifício residencial de arquitetura contemporânea ou quando uma edificação que antes era um tipo básico foi tão modificada que perdeu a maioria de suas características originais. Ambos os casos podem ser observados na Figura 08. Novamente, esse tipo tipológico aparece somente nos miolos de quadra. 

Revista Nacional de

Gerenciamento de Cidades

Outra tipologia comum na área estudada são os galpões, que podem dar lugar a várias lojas sob o mesmo telhado ou a uma única e grande loja, como é o caso dos galpões mostrados na figura 11. Alguns desses galpões são reminiscentes das estruturas existentes para dar suporte à Estação Ferroviária.

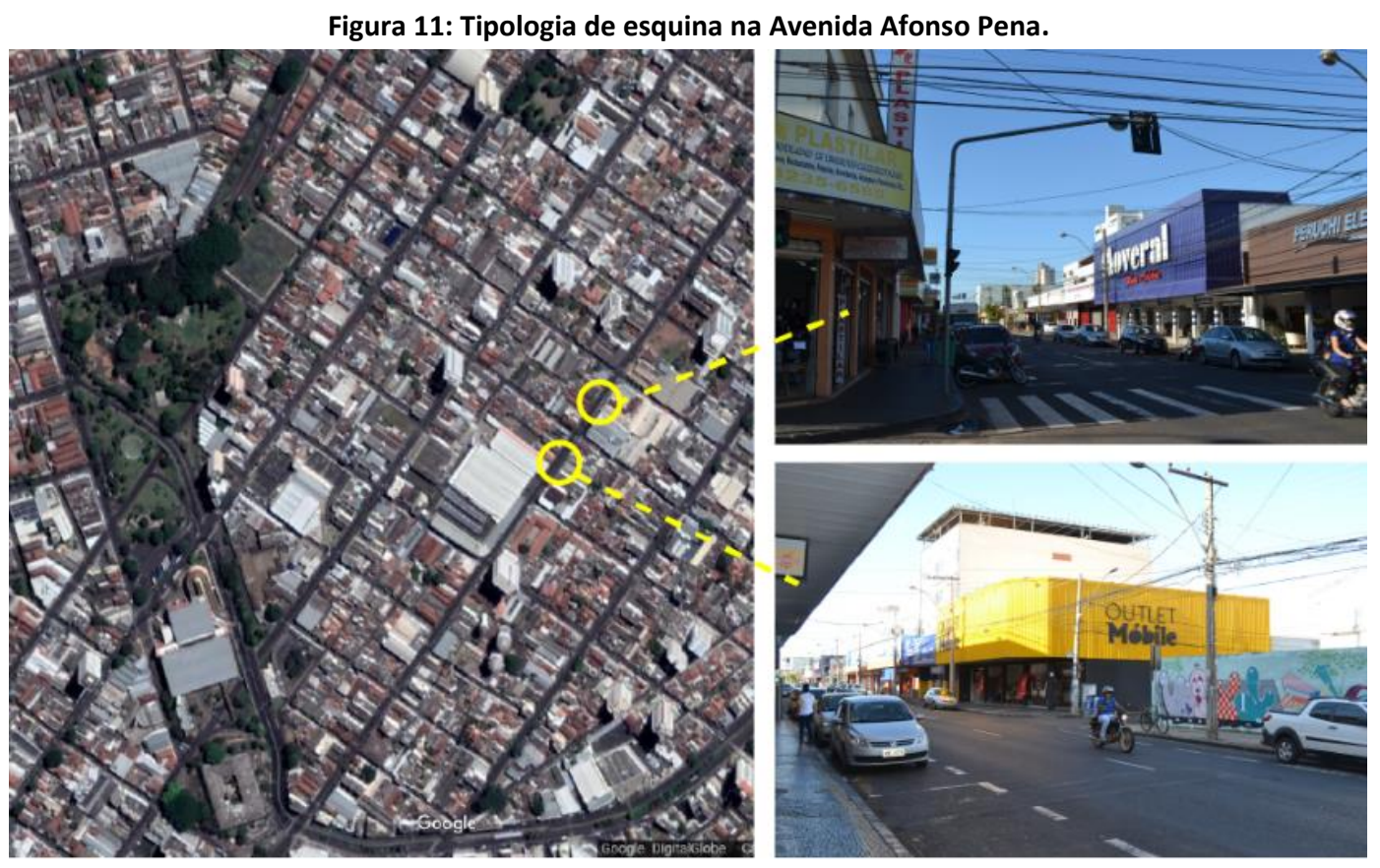

Fonte: Elaborada pelos autores, 2018.

Figura 12: Tipologias na Av. Afonso Pena.
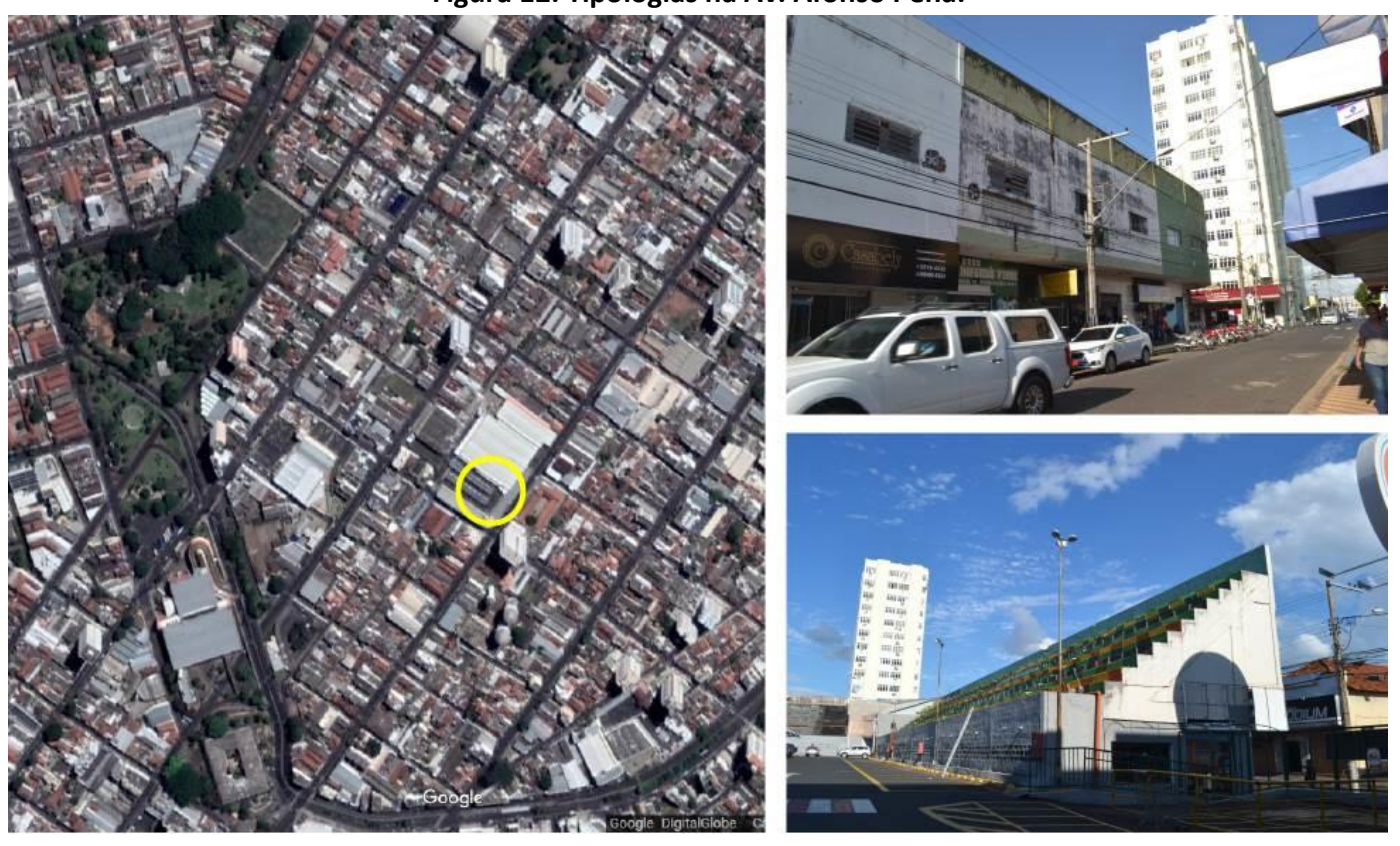


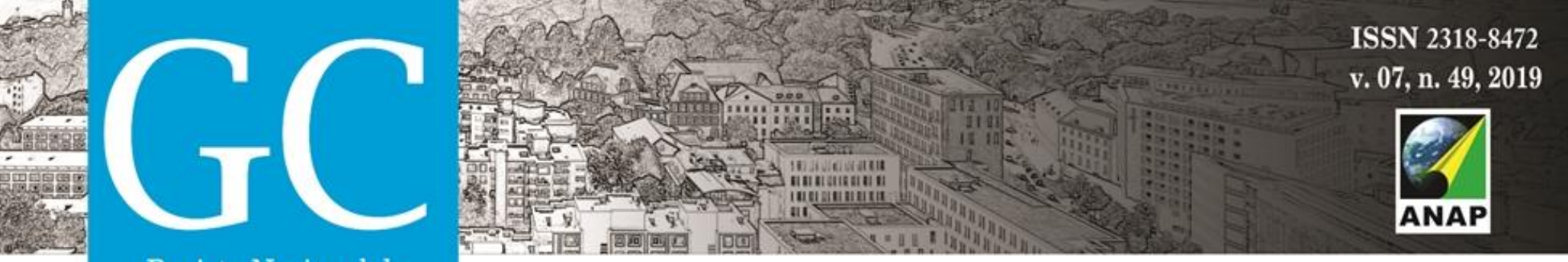

\section{Revista Nacional de}

Gerenciamento de Cidades

MOUDON, Anne Vernez. Getting to Know the Built Landscape: Typomorphology. In: FRANK, K. A. \& SCHNEEKLOTH (ed.). Ordering Space: Types in Architecture and Design, 1994

OLIVEIRA, Vitor (ed.). Diferentes abordagens da forma urbana. Contribuições luso-brasileira. Urban Forms, 2018.

PEREIRA COSTA, Staël de Alvarenga; GIMMLER NETTO, Maria Manoela. Fundamentos de morfologia urbana. Belo Horizonte: C/Arte, 2015.

PEGORARO, Rafael Lopez. Transformação Urbana no Brasil: Estudo de Cinco Centros Urbanos. Relatório Ciêntífico. São Paulo, 2016 